\title{
Status and schedule of SuperKEKB
}

\section{Kyo Shibata ${ }^{1}$}

High Energy Accelerator Research Organization (KEK)

1-1 Oho, Tsukuba-shi, Ibaraki-ken 305-0801, Japan

E-mail: kyo.shibata@kek.jp

SuperKEKB, which is an upgrade of the KEKB B-factory (KEKB), is a next-generation highluminosity electron-positron collider with asymmetric energies of $7 \mathrm{GeV}(\mathrm{e}-)$ and $4 \mathrm{GeV}(\mathrm{e}+$ ). Its design luminosity is $8.0 \times 10^{35} \mathrm{~cm}^{-2} \mathrm{~s}^{-1}$, which is about 40 times than the KEKB's record, and it is expected that the total integrated luminosity will reach $50 \mathrm{ab}^{-1}$ just over ten years after inauguration. To achieve this challenging goal, bunches of both beams are squeezed extremely to the nanometer scale $(0.3 \mathrm{~mm}$ across and $100 \mathrm{~nm}$ high), and the beam currents are doubled. To realize this, many upgrades must be performed, such as the replacement of magnets and beam pipes, construction of a damping ring for positrons and upgrade of the injector linac and RF systems. The dismantlement of KEKB and construction of SuperKEKB started on July 2010, and they are still underway. The commissioning will start in the second half of the Japanese fiscal year of 2014 .

The 2011 Europhysics Conference on High Energy Physics, EPS-HEP 2011,

Grenoble, Rhône-Alpes France

July 21-27 2011

\footnotetext{
${ }^{1}$ Speaker.
} 


\section{Introduction}

SuperKEKB $[1,2]$, which is an upgrade of the KEKB B-factory (KEKB), is a nextgeneration high-luminosity electron-positron collider with asymmetric energies of $7 \mathrm{GeV}$ (e-) and $4 \mathrm{GeV}\left(\mathrm{e}^{+}\right)$. Its predecessor, KEKB, was operated from 1998 to 2010 and had been a leader in the race to provide the world's highest luminosity since 2001. It delivered a total integrated luminosity of more than $1 \mathrm{ab}^{-1}$ to the Belle detector and made a great contribution to confirmation of $\mathrm{CP}$ violation in the neutral $\mathrm{B}$ meson system. To pursue research on flavor physics, however, much more luminosity is required and the SuperKEKB project was begun last year (2010). SuperKEKB is constructed on the site of the KEKB, and many facilities and components of KEKB, such as the tunnel, buildings, magnets and RF cavities, are reused to reduce the construction costs. The main upgrade is the positron ring (LER), and the electron ring (HER) is left as it is for the most part because it was found that the present cells in HER can be reused at SuperKEKB.

At the SuperKEKB project, a 50-fold increase in integrated luminosity is expected just over ten years after inauguration. The design luminosity is $8.0 \times 10^{35} \mathrm{~cm}^{-2} \mathrm{~s}^{-1}$, which is about 40 times than the KEKB's record. In this paper, how to achieve this challenging goal at SuperKEKB and major items to be upgraded are discussed. The status and schedule of the upgrade are also provided.

\section{Design concept of SuperKEKB}

When the beam sizes and the beta functions of both beams are equal at the interaction point (IP), the luminosity is expressed by the following formula:

$$
L=\frac{\gamma_{ \pm}}{2 e r_{\mathrm{e}}}\left(1+\frac{\sigma_{\mathrm{y}}^{*}}{\sigma_{\mathrm{x}}^{*}}\right)\left(\frac{I_{ \pm} \xi_{\mathrm{y} \pm}}{\beta_{\mathrm{y} \pm}^{*}}\right)\left(\frac{R_{\mathrm{L}}}{R_{\xi_{\mathrm{y}}}}\right) \propto \frac{I_{ \pm} \xi_{\mathrm{y} \pm}}{\beta_{\mathrm{y} \pm}^{*}},
$$

where $\gamma_{ \pm}$is the Lorentz factor, $e$ is the elementary electric charge, $r_{\mathrm{e}}$ is the classical electron radius, $\sigma_{\mathrm{x}, \mathrm{y}}{ }^{*}$ is the beam size in the horizontal and vertical directions at the IP, $I$ is the beam current, $\xi_{\mathrm{y} \pm}$ is the vertical beam-beam parameter, $\beta_{\mathrm{y} \pm}{ }^{*}$ is the vertical beta function at the IP, $R_{\mathrm{L}}$ and $R_{\xi y}$ are the reduction factors for the luminosity and the vertical beam-beam tune-shift parameter, respectively, owing to the crossing angle and the hourglass effect. The subscripts + and - indicates a positron or electron, respectively. In order to increase the luminosity, there are three choices: increasing $I_{ \pm}$and $\xi_{\mathrm{y} \pm}$ and squeezing $\beta_{\mathrm{y} \pm}{ }^{*}$. However, it was found at the KEKB that it is not easy to increase $\xi_{\mathrm{y} \pm}$; consequently, at SuperKEKB it remains the same as at the KEKB.

On the other hand, crossing the beam by using the "nanobeam scheme" [3] makes it possible to squeeze $\beta_{\mathrm{y} \pm}{ }^{*}$ to about $1 / 20$ th of its original size. In the nanobeam scheme, the bunches of both beams are squeezed extremely to the nanometer scale $(0.3 \mathrm{~mm}$ across and 100 $\mathrm{nm}$ high) and intersect only in the highly focused region of each bunch at a large crossing angle (4.8 degrees) as shown in Fig.1. In the conventional collision scheme, $\beta_{\mathrm{y}}{ }^{*}$ must be larger than the bunch length $\left(\sigma_{\mathrm{z}}\right)$, which is the so-called hourglass requirement $\left(\beta_{\mathrm{y}}^{*} \geq \sigma_{\mathrm{z}}\right)$ [4]. However, in the nanobeam scheme, it is possible to squeeze $\beta_{\mathrm{y}}{ }^{*}$ to a size much less than the bunch length 
because the overlap region is very small and $\sigma_{\mathrm{x}}{ }^{*} / \phi(\approx 300 \mu \mathrm{m}, \phi$ : half crossing angle) is substituted for $\sigma_{\mathrm{z}}$ $(\approx 6 \mathrm{~mm})$. Since a small $\sigma_{\mathrm{x}}^{*}$ is necessary in this collision scheme, it is required to realize a low horizontal emittance $\left(\varepsilon_{\mathrm{x}}\right)$ and small horizontal beta function at the IP
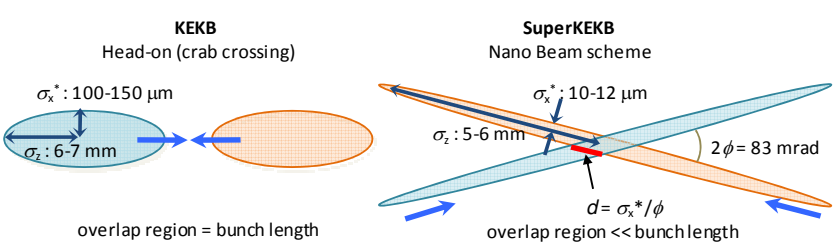

Figure 1: Schematic view of beam collision scheme of KEKB and SuperKEKB.

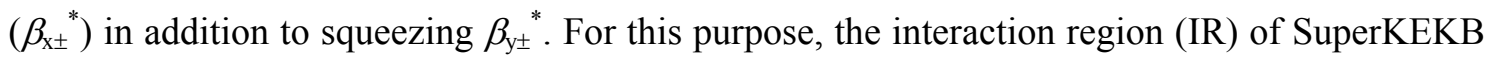
will be changed drastically, and new superconducting magnets for final focusing will be installed deeper in the IR. Moreover, to have low-emittance beams, construction of a damping ring for positrons, replacement of magnets, precise alignment of the magnets, etc. will also be required. To mitigate the emittance growth due to intra-beam scattering, the beam energy of the LER will be increased from 3.5 to $4.0 \mathrm{GeV}$, which will also be effective in lengthening the Touschek lifetime. This collision scheme should produce 20 times the luminosity produced in the KEKB.

The luminosity will also be doubled by increasing the beam current to $2.6 \mathrm{~A}$ (e-) and $3.6 \mathrm{~A}$ $(\mathrm{e}+)$, which are twice as much as in the KEKB. To achieve this, almost all beam pipes of the LER will be replaced with new ones with antechambers that can deal with the unfortunate side effects of a high-beam current in the positron ring (namely, the electron-cloud effect [5]) as well as excessive heating in the beam pipe due to the strong synchrotron radiation (SR) [6]. On the other hand, most of the beam pipes of the HER will be reused. Because the beam energy of the electron is reduced from 8.0 to $7.0 \mathrm{GeV}$, the power of the SR decreases to the tolerance level of a conventional copper beam pipe, in spite of doubling the beam current. Additionally, other modifications are also necessary, such as upgrades of the positron source and RF systems.

\section{Major items in upgrade}

The outline of the upgrade to SuperKEKB is shown in Fig. 2 , and the main parameters of the KEKB and SuperKEKB are summarized in Table 1. In this section, the major items to be upgraded, the magnets, beam pipes, IR, RF system, injector linac, and beam transport system, are discussed.

In order to reduce the

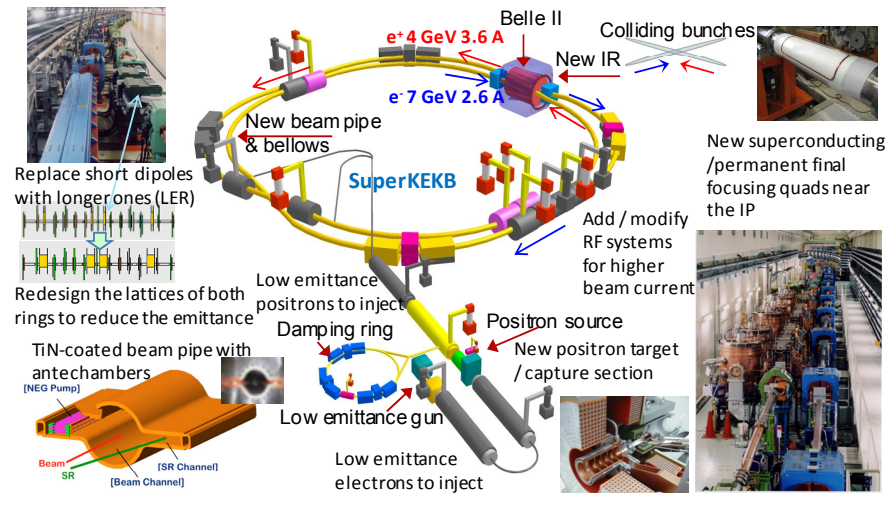

Figure 2: Outline of upgrade to SuperKEKB. horizontal emittance to $1 / 5-1 / 10$ of KEKB's values, a large number of magnets need to be rearranged, replaced, and added. In the LER arc section, about 100 bending magnets with a length of $0.89 \mathrm{~m}$ are being replaced with new longer ones $(4.2 \mathrm{~m})$. In the LER wiggler section, 56 new single pole wigglers and 112 half pole wigglers will be added to the existing normal 
Table 1: Machine parameters of KEKB (LER/HER) and SuperKEKB (LER/HER)

\begin{tabular}{l|c|c|c|c}
\hline & KEKB design & KEKB Achieved: with crab & SuperKEKB & Unit \\
\hline \hline Energy & $3.5 / 8.0$ & $3.5 / 8.0$ & $4.0 / 7.0$ & $\mathrm{GeV}$ \\
$\beta_{\mathrm{y}}{ }^{*}$ & $10 / 10$ & $5.9 / 5.9$ & $0.27 / 0.30$ & $\mathrm{~mm}$ \\
$\beta_{\mathrm{x}}{ }^{*}$ & $330 / 330$ & $1200 / 1200$ & $32 / 25$ & $\mathrm{~mm}$ \\
$\varepsilon_{\mathrm{x}}$ & $18 / 18$ & $18 / 24$ & $3.2 / 5.3$ & $\mathrm{~nm}$ \\
$\mathrm{x}-\mathrm{y}$ coupling $\left(\varepsilon_{\mathrm{y}} / \varepsilon_{\mathrm{x}}\right)$ & 1 & $0.85 / 0.64$ & $0.27 / 0.24$ & $\%$ \\
$\sigma_{\mathrm{y}}{ }^{*}$ & 1.9 & 0.94 & $0.048 / 0.062$ & $\mu \mathrm{m}$ \\
$\xi_{\mathrm{y}}$ & 0.052 & $0.129 / 0.090$ & $0.09 / 0.081$ & $\mathrm{~mm}$ \\
$\sigma_{\mathrm{z}}$ & 4 & $6-7$ & $6 / 5$ & $\mathrm{~A}$ \\
$I$ & $2.6 / 1.1$ & $1.64 / 1.19$ & $3.6 / 2.6$ & 2500 \\
$N_{\text {bunch }}$ & 5000 & 1584 & 80 & $10^{34} \mathrm{~cm}^{-2} \mathrm{~s}^{-1}$ \\
\hline Luminosity & 1 & 2.11 & &
\end{tabular}

ones to double the wiggler cycles. Regarding the quadrupole and sextupole magnets, most of them will be reused and rearranged. On the other hand, in the HER, the wiggler section will be newly made to reduce the emittance. For 6 of the 10 wiggler sections, the present LER wiggler magnets will be reused. Most of the other magnets of the HER will be left as they are.

The beam pipes in the LER arc section are being replaced with new aluminum-alloy pipes with antechambers (Fig. 3) to cope with the electron cloud issue and heating problem. The length of the replacement is about $2000 \mathrm{~m}$. On the other hand, in the HER arc section, the present copper beam pipes will be reused without any modification. Since the HER energy will be reduced from 8.0 to $7.0 \mathrm{GeV}$, the SR power at the normal arc section will be roughly the same as in the KEKB, even though the beam current will be doubled. In wiggler section of both rings, copper beam pipes with antechambers are newly installed because the SR power is much larger than that in the arc section, and the aluminum-alloy pipe can not withstand it.

Several types of countermeasures will be adopted in the LER to deal with the electron cloud issues. In the wiggler section, electrons will be attracted by the clearing electrode [7], which will be mounted on the inner surface of the beam pipe. On the other hand, in the bending magnet, the effective secondary electron yield (SEY) will be structurally reduced by the groove surface [8] with a TiN coating [9], from which the secondary electrons can hardly escape especially in the dipole field, as shown in Fig. 3. In the draft space, the electron cloud will be mitigated by the TiN coating and a conventional solenoid field.

The IR will be changed drastically in order to adopt the new collision scheme. The final superconducting quadrupole magnets are located as close to the IP as possible to squeeze $\beta_{\mathrm{y} \pm}{ }^{*}$ extremely. Independent magnets in the LER and HER will be used. The design work on the IR has not yet been completed. The optics design, local chromaticity correction scheme, hardware design, assembly procedure, and evaluation of background issues are also now under discussion. They will be fixed within this year.

Regarding the RF system, since the beam power required for the HER and
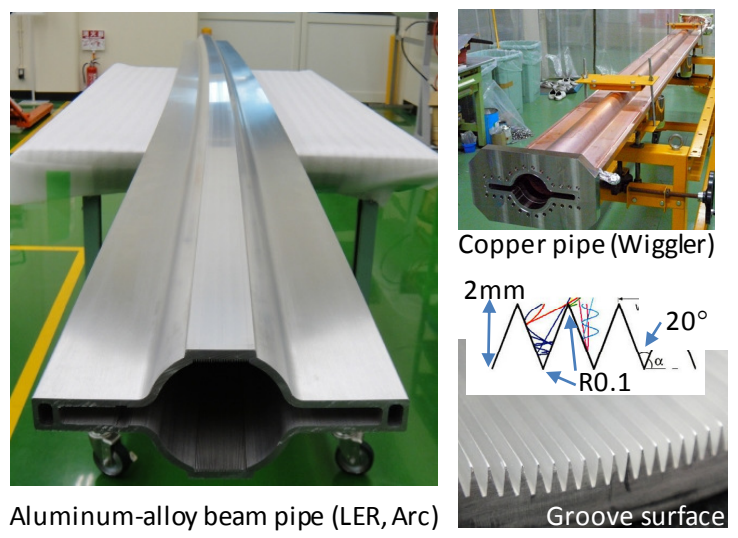

Figure 3: Beam pipes of SuperKEKB and the groove surface as a countermeasure against electron cloud issues. 
LER are 1.5 and 2.5 times higher than that required for the KEKB, respectively, it is necessary to increase the beam power per cavity at SuperKEKB. For the normal conducting cavity (ARES), the number of RF stations where one klystron feeds one cavity will be increased while one klystron typically feeds two cavities in the case of the KEKB. Moreover, additional HP and LL RF systems, rearrangement of cavities, and replacement of the input couplers with larger ones are also needed. The improvement of the HOM dampers is not necessary for only ARES but also for superconducting cavities.

Upgrades to the injector linac and beam transport system are being designed to improve the rate and quality of injected beams to deliver the required beams with increased injection efficiencies [10]. The main upgrades to be made are a new low-emittance RF electron gun, improvement of the positron source, and installation of a $1.1 \mathrm{GeV}$ positron damping ring [11].

Besides the above items, there are many other upgrades such as improvement of the beam monitor [12] and control system, reinforcement of the cooling system for the magnet and vacuum system, construction of a tunnel and buildings for the damping ring.

\section{Status and schedule}

The construction schedule is summarized in Fig. 4. The total budget for upgrade is 31.4 billion yen and it will be executed between the Japanese fiscal years (JFY) 2010 and 2014. The Operating budget is expected in the JFY2014 and later. This year (2011) the dismantlement of the KEKB, mass fabrication of magnets and beam pipes for SuperKEKB, etc. are underway.

Dismantlement work on the KEKB started in July 2010 and was going smoothly until March 11 $11^{\text {th }}$ 2011, when a huge earthquake hit eastern Japan. At the KEKTsukuba site, some infrastructures and

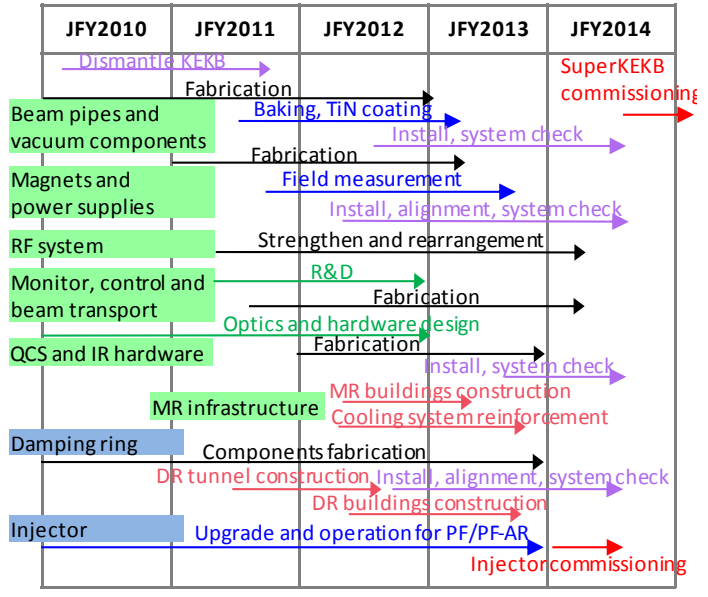

Figure 4: Construction schedule. components of accelerators were damaged even though it was some $300 \mathrm{~km}$ away from the epicenter. The damage to the KEKB main rings appears to be less serious, though nonnegligible. An air fin cooler for klystrons, RF control system, equipment for beam monitoring, etc. were founded to be damaged. Regarding the tunnel, the distortion due to the earthquake made large gaps of up to $4 \mathrm{~mm}$ at the expansion joint, which might require the survey and realignment of magnets and reference points. For the dismantlement effort, however, it will be possible to eliminate 1.5 months of delay within a certain time frame of the construction; the schedule has not changed so far.

Commissioning of the main ring and damping ring will start in the second half of the JFY 2014. The injector linac is in operation for synchrotron radiation facilities during the construction period, and test operation for the upgrade will be performed in parallel. Commissioning of the linac for SuperKEKB will start in the beginning of the JFY2014. On the 
other hand, it is required by the detector group that the machine operation starts without the Belle II detector. So, the machine commissioning will start with some dummy chambers with a luminosity monitor at the IR, the so-called BEAST. IR configuration, before Belle II roll-in, is under discussion now. Figure 5 shows the projection of the integrated luminosity of SuperKEKB. It is expected that the total integrated luminosity will reach $50 \mathrm{ab}^{-1}$ about ten

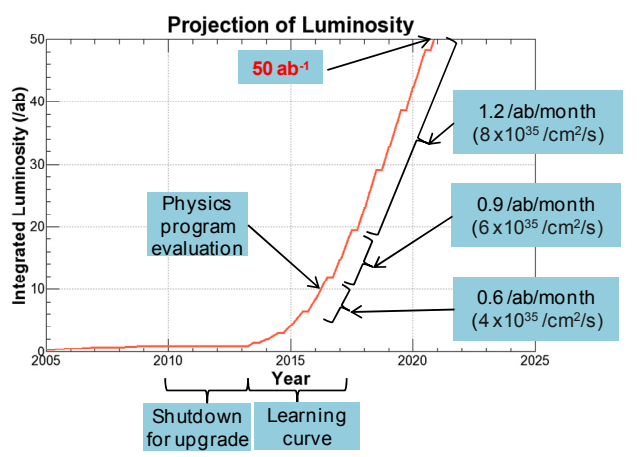

Figure 5: Projection of luminosity. years after inauguration.

\section{Acknowledgment}

The author would to thank colleagues on the SuperKEKB team.

\section{References}

[1] SuperKEKB WWW pages, http://www-superkekb.kek.jp/

[2] T. Abe, et al., Belle II Technical Design Report, [physics / 1011.0352]

[3] P. Raimondi, NEW DEVELOPMENTS IN SUPER B-FACTORIES, Proceedings of PAC'07 (2007) p.32.

[4] M. A. Furman, Hourglass Effects for Asymmetric Colliders, Proceedings of PAC'91 (1991) p.422.

[5] K. Ohmi et al., Head-Tail Instability Caused by Electron Clouds in Positron Storage Rings, Phys. Rev. Lett. 85 (2000) p.3821.

[6] Y. Suetsugu, et al., Development of copper beam ducts with antechambers for advanced highcurrent particle storage rings, Vacuum 84 (2010) p.694

[7] Y. Suetsugu, et al., Demonstration of electron clearing effect by means of a clearing electrode in high-intensity positron ring, MIN-PR-A 598 (2009) p. 372

[8] Y. Suetsugu, et al., Continuing study o electron-cloud clearing techniques in high-intensity positron ring: Mitigation by using groove surface in vertical magnetic field, MIN-PR-A 604 (2009) p.449

[9] K. Shibata, et al., DEVELOPMENT OF TiN COATING SYSTEM FOR BEAM DUCTS OF KEK B-FACTORY, Proceedings of EPAC'08 (2008) p.1700

[10] T. Kamitani, et al., INJECTOR LINAC UPGRADE FOR SuperKEKB, Proceedings of LINAC'10 (2010) p.70.

[11] M. Kikuchi, et al., DESIGN OF POSITRON DAMPING RING FOR SUPER-KEKB, Proceedings of IPAC'10 (2010) p.1641.

[12] M. Tobiyama, et al., DEVELOPMENT OF BUTTON ELECTRODES FOR SUPERKEKB RINGS, Proceedings of BIW'10 (2010) p.223. 